\title{
Compressibility effects on homogeneous isotropic turbulence using Schur decomposition of the velocity gradient tensor
}

\author{
R. Boukharfane $*$, A. Er-raiy ${ }^{\dagger}$ and M. Parsani ${ }^{\ddagger}$ \\ King Abdullah University of Science and Technology (KAUST), Computer Electrical and Mathematical Science and \\ Engineering Division (CEMSE), Extreme Computing Research Center (ECRC), 23955-6900, Thuwal, Saudi Arabia
}

The study of compressibility effects on the dynamics and the structure of turbulence is an important, but difficult, topic in turbulence modeling. Taking advantage of a recently proposed Schur decomposition approach (Keylock, C. J., The Schur decomposition of the velocity gradient tensor for turbulent flows, Journal of Fluid Mechanics, 2018) to decompose the velocity gradient tensor into its normal and non-normal parts, here we evaluate the influence of the compressibility on some statistical properties of the turbulent structures. We perform a set of direct numerical simulations of decaying compressible turbulence at six turbulent Mach numbers between $\mathrm{M}_{t}=0.12$ and $\mathrm{M}_{t}=0.89$ and a Reynolds number based on the Taylor micro-scale of $\operatorname{Re}_{\lambda}=100$. All the simulations have been carried out using an improved seventhorder accurate WENO scheme to discretize the non-linear advective terms and an eight-order accurate centered finite difference scheme is retained for the diffusive terms. In the double decomposition, the normal parts of the velocity gradient tensor (represented by the eigenvalues) are separated explicitly from non-normal components. The two-dimensional space defined by the second and third invariants of the velocity gradient tensor is subdivided into six regions and the contribution of each regional term to the Schur decomposition of the velocity gradient tensor is analyzed. Our preliminary findings show the difficulty of understanding the nonlocal effects without taking into account both the normal contribution (represented by the eigenvalues) and the non-normal component computed with of the Schur decomposition.

\section{Introduction}

$\mathrm{T}$ URBULENCE factors into the design of much of our technology, from airplanes, cars, ships, pipelines, reactors, and wind turbines. Turbulence is also a key element for predicting important natural phenomena such as the weather and oceans circulation. The concurrence of many linear and nonlinear processes in combination with the typical large spectra of time and length scales makes turbulence a very challenging problem to comprehend. To consider turbulence a solved problem in physics, we would need to be able to demonstrate that we can start with the basic equation describing fluid motion (a mathematical object that in many aspects is still not completely defined) and then solve it to predict,

\footnotetext{
* Postdoctoral research fellow, radouan.boukharfane@ kaust.edu.sa, AIAA Member

†Postdoctoral research fellow, aimad.erraiy@kaust.edu.sa, AIAA Member

$\doteqdot$ Assistant Professor, matteo.parsani@kaust.edu.sa, AIAA Member
} 
in detail, how a fluid will move under any particular set of conditions. That cannot be achieved and, therefore, many physicists consider turbulence to be an unsolvable problem.

However, we know that understanding velocity gradient dynamics and small-scale structure offer an interesting insight into turbulence mechanisms such as energy cascade, mixing, material element deformation, vortex stretching, and intermittency. Analyzing the local motion by means of the eigenvalues and the invariants of the velocity gradient tensor, $\mathcal{A}$ with components $\mathcal{A}_{i j}=\partial \mathcal{U}_{i} / \partial x_{j}, i, j=1,2,3$, is often used to to enhance our understanding of the dynamics of the flow structure topologies, distortion, and their interaction with dissipation [1-3]. Such analysis allows us to develop dynamical models for describing the small-scale behavior that in turn, provides better prospects for practical modeling of turbulent flows.

The general pattern of the three-dimensional streamlines in the immediate vicinity of an observer moving with any point in the flow field can be determined by looking at the nature of the eigenvalues, $\left(\lambda_{1}, \lambda_{2}, \lambda_{3}\right)$, of the velocity gradient tensor evaluated at the point. Such eigenvalues are the roots of the characteristic equation of $\mathcal{A}_{i j}$ which can be written as

$$
\lambda^{3}+P^{\mathcal{A}} \lambda^{2}+Q^{\mathcal{A}} \lambda^{2}+R^{\mathcal{A}}=0
$$

where $P^{\mathcal{A}}, Q^{\mathcal{A}}$, and $R^{\mathcal{A}}$ are the invariants of the velocity gradient tensor,

$$
\begin{aligned}
P^{\mathcal{A}} & =-\sum_{i=1}^{3} \lambda_{i} \equiv-\mathcal{S}_{i i}^{\mathcal{A}}, \\
R^{\mathcal{A}} & =\sum_{i<j}^{3} \lambda_{i} \lambda_{j} \equiv \frac{1}{2}\left(\left(P^{\mathcal{A}}\right)^{2}-\mathcal{S}_{i j}^{\mathcal{A}} \mathcal{S}_{j i}^{\mathcal{A}}+\Omega_{i j}^{\mathcal{A}} \Omega_{j i}^{\mathcal{A}}\right) \\
Q^{\mathcal{A}} & =\prod_{i=1}^{3} \lambda_{i} \equiv \frac{1}{3}\left(-\left(P^{\mathcal{A}}\right)^{3}+3 P^{\mathcal{A}} Q^{\mathcal{A}}-\mathcal{S}_{i j}^{\mathcal{A}} \mathcal{S}_{j k}^{\mathcal{A}} \mathcal{S}_{k i}^{\mathcal{A}}-3 \Omega_{i j}^{\mathcal{A}} \Omega_{j k}^{\mathcal{A}} \mathcal{S}_{k i}^{\mathcal{A}}\right)
\end{aligned}
$$

In the above definition, the symbol $\mathcal{S}_{i j}^{\mathcal{A}}=\left(\mathcal{A}_{i j}+\mathcal{A}_{j i}\right) / 2$ indicates the $i j$ component of the symmetric strain-rate tensor, $\mathcal{S}^{\mathcal{A}}$, whereas $\Omega_{i j}^{\mathcal{A}}=\left(\mathcal{A}_{i j}-\mathcal{A}_{j i}\right) / 2$ represents the $i j$ component of the the skew-symmetric rotation-rate tensor, $\mathbf{\Omega}^{\mathcal{A}}$. The discriminant surface, $\Delta^{\mathcal{A}}$, is given by

$$
\Delta^{\mathcal{A}}=27\left(R^{\mathcal{A}}\right)^{2}+\left(4\left(P^{\mathcal{A}}\right)^{2}-18 P^{\mathcal{A}} Q^{\mathcal{A}}\right) R^{\mathcal{A}}+\left(4\left(Q^{\mathcal{A}}\right)^{3}-\left(P^{\mathcal{A}}\right)^{2}\left(Q^{\mathcal{A}}\right)^{2}\right)
$$

and the tent-like curve in fig. 1 is the curve along which the discriminant of the velocity gradient tensor is equal to zero. This curve separates focal from non-focal local topologies of the joint probability density function (PDF) of the space $Q^{\mathcal{A}}-R^{\mathcal{A}}$. Such space contains substantial information on turbulence. For an incompressible flow, we can distinguish in the $Q-R$ space six non-degenerate topology types (c.f. fig.11):

- $\Delta^{\mathcal{A}}>0, R^{\mathcal{A}}>0\left(Q^{\mathcal{A}}>0\right.$ for region 1 and $Q^{\mathcal{A}}<0$ for region 5): compressing towards an unstable focus region (UFC). The difference between the two regions lies in the strength of the enstrophy; 
- $\Delta^{\mathcal{A}}>0, R^{\mathcal{A}}<0\left(Q^{\mathcal{A}}>0\right.$ for region 2 and $Q^{\mathcal{A}}<0$ for region 3): stretching away from a stable focus region (SFS). Similarly, the difference between the two regions lies in the strength of the enstrophy;

- $\Delta^{\mathcal{A}}<0, R^{\mathcal{H}}>0$ (region 6): two saddles points with an unstable node (UNSS);

- $\Delta^{\mathcal{A}}<0, R^{\mathcal{A}}<0$ (region 4): two saddles points with a stable node (SNSS).

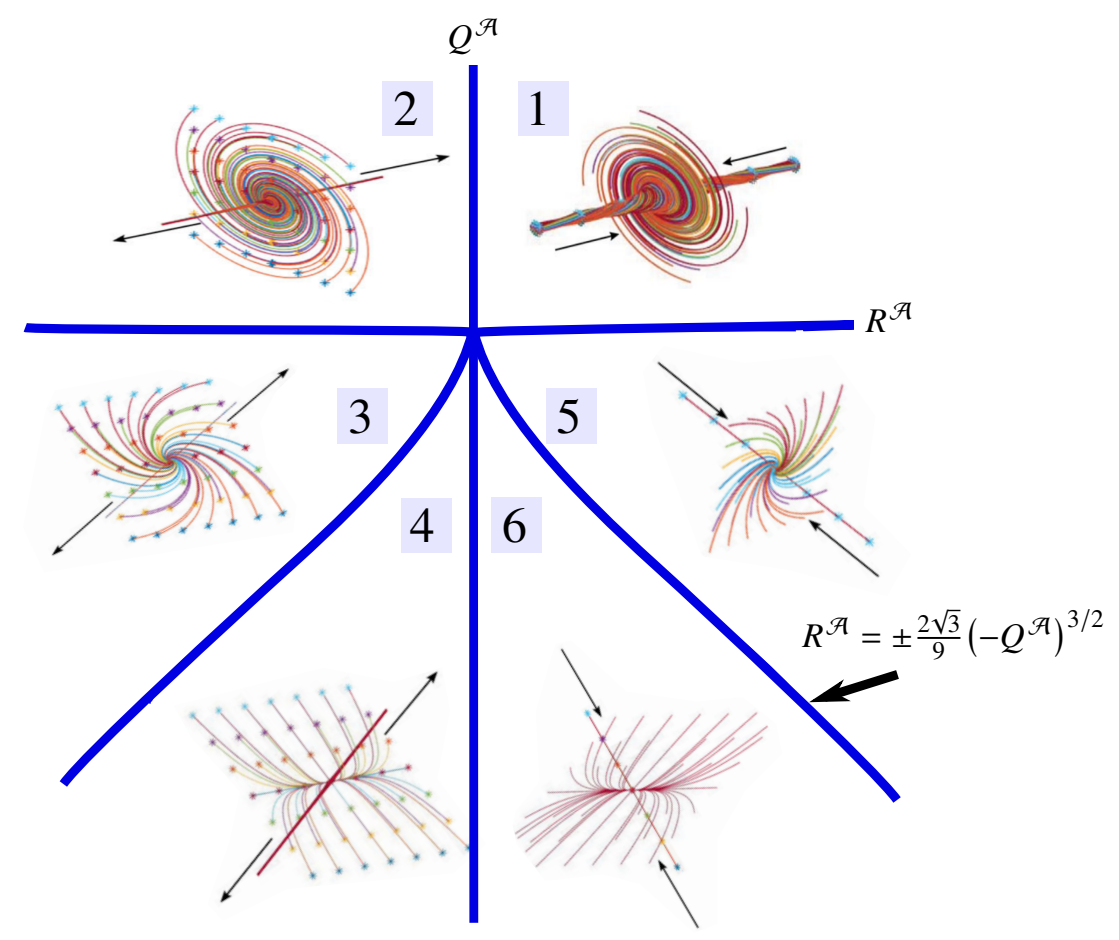

Fig. 1 Classification of the local three-dimensional streamlines into non-degenerate topologies in the $Q-R$ plane for incompressible turbulence [1, 4]. The solid lines indicate the discriminant, $\Delta^{\mathcal{A}}$, lines.

The second invariant, $Q^{\mathcal{A}}$, represents the difference between the enstrophy and the total strain, whereas the third invariant, $R^{\mathcal{A}}$, indicates the difference between the strain production and the enstrophy production. There are significant dynamical effects associated with $Q^{\mathcal{A}}$ and $R^{\mathcal{A}}$. However, it is still unclear how local and non-local effects contribute to these dynamics, and how these dynamics vary for different flows.

Conventional studies use the physically interpretable decomposition of the velocity gradient tensor into strain, $\mathcal{S}$, and rotation, $\boldsymbol{\Omega}$, components, $\mathcal{A}=\mathcal{S}+\boldsymbol{\Omega}$, with the latter often then expressed as a vorticity vector. However, the eigenvalues of the velocity gradient tensor are also important for delimiting different topological states of the tensor; see Keylock [5] and the references therein. In 2018, Keylock [5] proposed to use the Schur decomposition to separate explicitly the normal part of the tensor (represented by the eigenvalues) from the non-normality contributions, $\mathcal{A}=\mathcal{B}+C$ (characterizing the tensor asymmetries). Then, he used the Hermitian/skew-Hermitian decomposition's of $\mathcal{B}$ and $\mathcal{C}$ to understand the relative importance of strain and rotation for both the normal and non-normal parts of the tensor. In his work, Keylock [5] focused on the properties of the velocity gradient tensor for incompressible, homogeneous, isotropic turbulence. Here, we extend his work to compressible flows. To this end, we evaluate the compressibility 
effects on some statistical properties of the turbulent structures of the compressible, homogeneous, isotropic turbulence. We perform a set of direct numerical simulations of decaying compressible turbulence at six turbulent Mach numbers between $\mathrm{M}_{t}=0.12$ and $\mathrm{M}_{t}=0.89$ and a Reynolds number based on the Taylor micro-scale of $\operatorname{Re}_{\lambda}=100$.

\section{Schur decomposition-based of the velocity gradient tensor}

The general (complex) Schur decomposition [6] applied to a general complex matrix, $\mathcal{M} \in \mathbb{C}^{n \times n}$, is given by

$$
\mathcal{M}=\mathfrak{U} \mathfrak{T}^{*},
$$

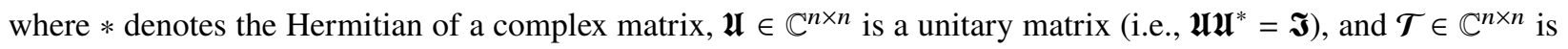
upper-triangular. Thus, the characteristic polynomials of $\mathcal{M}$ and $\mathcal{T}$ are the same and the roots for the latter are simply the diagonal elements of $\mathcal{T}$. This decomposition is valid for any complex or real matrix. The matrix $\mathcal{T}$ in $(6)$ is given by

$$
\mathcal{T}=\left(\begin{array}{ccccc}
\lambda_{1} & \Lambda_{12} & \Lambda_{13} & \cdots & \Lambda_{1, n} \\
0 & \lambda_{2} & \Lambda_{23} & \cdots & \Lambda_{2, n} \\
\vdots & \vdots & \vdots & \ddots & \vdots \\
0 & 0 & 0 & 0 & \lambda_{n}
\end{array}\right)=\left(\begin{array}{ccccc}
\mathcal{L}_{11} & \Lambda_{12} & \Lambda_{13} & \cdots & \Lambda_{1, n} \\
0 & \mathcal{L}_{22} & \Lambda_{23} & \cdots & \Lambda_{2, n} \\
\vdots & \vdots & \vdots & \ddots & \vdots \\
0 & 0 & 0 & 0 & \mathcal{L}_{n n}
\end{array}\right)=\mathcal{L}+\boldsymbol{\Lambda}
$$

where $\mathcal{L}$ is a diagonal matrix whose elements correspond to the eigenvalues of $\mathcal{M},\left(\lambda_{1}, \lambda_{2}, \ldots, \lambda_{n}\right)=\left(\mathcal{L}_{11}, \mathcal{L}_{22}, \ldots, \mathcal{L}_{n n}\right)$, and $\boldsymbol{\Lambda}$ is an upper triangular matrix that represents the non-normal part of $\mathcal{M}$ (characterizing the matrix asymmetries). Following Keylock [5], we first wish to decompose the velocity gradient tensor, $\mathcal{A} \in \mathbb{R}^{3 \times 3}$, into the normal, $\mathcal{B}$, and non-normal, $\mathcal{C}$, contributions:

$$
\mathcal{A}=\mathcal{B}+\mathcal{C} .
$$

The tool from linear algebra used to accomplish this is the complex Schur transform (6). To ensure the uniqueness of the decomposition of $\mathcal{A}$, the ordering of the eigenvalues of $\mathcal{A}$ is fixed in a increasing order, i.e., $\mathcal{L}_{11}$ (resp. $\mathcal{L}_{33}$ ) represents the most negative (resp. the most positive) eigenvalue of $\mathcal{A}$. Therefore, by applying the transformation (6) to $\mathcal{A}$ we get following expressions

$$
\begin{aligned}
\mathcal{B} & =\mathfrak{U} \mathcal{L} \mathfrak{U}^{*}, \\
C & =\mathfrak{U} \mathbf{\Lambda} \mathfrak{U}^{*}
\end{aligned}
$$

Thus, we note that $\mathcal{B}$ acts locally and is related to the dynamics driven by the eigenvalues of $\mathcal{A}$, and $\mathcal{C}$ is associated with the vortical structures and the non-local effects of flow fields [5]. In order words, this decomposition explicitly gives i) the information related to the eigenvalues of $\mathcal{A}$ (which are associated with the local dynamics), and ii) the non-symmetric structure in $\mathcal{A}$ (which are induced by non-local effects). This information is not included in the 
conventional decomposition of $\mathcal{A}$ into its symmetric, $\mathcal{S}$, and skew-symmetric, $\boldsymbol{\Omega}$, parts,

$$
\mathcal{A}=\mathcal{S}+\boldsymbol{\Omega}
$$

Note that the non-normality could be measured by means of either $\left\|\mathcal{A} \mathcal{A}^{*}-\mathcal{A}^{*} \mathcal{A}\right\|$ or $\|\Lambda\|$, where $\|\phi\|=\sqrt{\operatorname{tr}\left(\phi \phi^{*}\right)} \equiv$ $\sqrt{\sum_{i, j=1}^{3}\left|\phi_{i j}\right|^{2}}$ is the Frobenius norm of a generic quantity $\phi$. The following concepts and formula, which associate the Schur decomposition components with the invariants of $\mathcal{A}$, are detailed in the recent work of Keylock [5]. As a second step, we take the strain-rate tensor and the rotation-rate tensor of $\mathcal{B}$ and $\boldsymbol{C}$. The resulting expressions are

$$
\left\{\begin{array}{l}
\mathcal{B}=\mathcal{S}^{\mathcal{B}}+\mathbf{\Omega}^{\mathcal{B}} \\
C=\mathcal{S}^{C}+\boldsymbol{\Omega}^{C}
\end{array}\right.
$$

where

$$
\left\{\begin{array}{l}
\mathcal{S}^{\mathcal{B}}=\frac{1}{2}\left(\mathcal{B}+\mathcal{B}^{*}\right) \\
\boldsymbol{\Omega}^{\mathcal{B}}=\frac{1}{2}\left(\mathcal{B}-\mathcal{B}^{*}\right)
\end{array}\right.
$$

and

$$
\left\{\begin{array}{l}
\mathcal{S}^{C}=\frac{1}{2}\left(C+C^{*}\right), \\
\mathbf{\Omega}^{C}=\frac{1}{2}\left(C-C^{*}\right) .
\end{array}\right.
$$

In this case, the Frobenius norm of the strain and rotation tensors read

$$
\begin{gathered}
\left\|\boldsymbol{S}^{\mathcal{A}}\right\|^{2}=\left\|\mathcal{S}^{\mathcal{B}}\right\|^{2}+\left\|\mathcal{S}^{C}\right\|^{2} \equiv\left\|\mathcal{S}^{\mathcal{B}}\right\|^{2}+\left\|\boldsymbol{\Omega}^{C}\right\|^{2} \\
\left\|\boldsymbol{\Omega}^{\mathcal{A}}\right\|^{2}=\left\|\boldsymbol{\Omega}^{\mathcal{B}}\right\|^{2}+\left\|\boldsymbol{\Omega}^{C}\right\|^{2} \equiv\left\|\boldsymbol{\Omega}^{\mathcal{B}}\right\|^{2}+\left\|\mathcal{S}^{C}\right\|^{2}
\end{gathered}
$$

Since the eigenvalues of $\mathcal{A}$ and $\mathcal{B}$ are identical, the invariants of the tensor $\mathcal{A}$ are identical to those of $\mathcal{B}$. Therefore, we have

$$
\left\{\begin{array}{l}
P^{\mathcal{B}}=P^{\mathcal{A}}=P^{\mathcal{S}^{\mathcal{B}}} \\
Q^{\mathcal{B}}=Q^{\mathcal{A}}=Q^{\mathcal{S}^{\mathcal{B}}}+Q^{\Omega^{\mathcal{B}}}=\frac{1}{2}\left(\left\|\boldsymbol{\Omega}^{\mathcal{B}}\right\|-\left\|\mathcal{B}^{\mathcal{B}}\right\|\right) \\
R^{\mathcal{B}}=R^{\mathcal{A}}=Q^{\mathcal{S}^{\mathcal{B}}}-E^{\mathcal{P}^{\mathcal{B}}}=-\operatorname{det}\left(\mathcal{S}^{\mathcal{B}}\right)-\operatorname{tr}\left(\left(\mathbf{\Omega}^{\mathcal{B}}\right)^{2} \mathcal{S}^{\mathcal{B}}\right),
\end{array}\right.
$$

and

$$
\left\{\begin{array}{l}
P^{C}=P^{\mathcal{S}^{C}}=P^{\Omega^{C}}=0 \\
Q^{C}=Q^{\mathcal{S}^{C}}+Q^{\Omega^{C}}=0 \\
R^{C}=Q^{\mathcal{S}^{C}}-E^{\mathcal{P}^{C}}=0
\end{array}\right.
$$


where $E^{\mathcal{P} \phi}=\frac{1}{4} \omega_{i} \mathcal{S}_{i j}^{\Phi} \omega_{j}$ is the enstrophy production rate for the generic quantity $\phi$. From the above identities, we note that the dynamics of $\mathcal{A}$ are only dictated by the normal part, $\mathcal{B}$. The second and third invariants contain non-normal contributions when $\|C\| \neq 0$. It is important to highlight that two new terms arise in both the strain and the enstrophy productions for the third invariant, i.e.,

$$
\begin{aligned}
-\operatorname{det}\left(\mathcal{S}^{\mathcal{A}}\right) & =-\operatorname{det}\left(\mathcal{S}^{\mathcal{B}}\right)+\operatorname{tr}\left(\left(\boldsymbol{\Omega}^{C}\right)^{2} \mathcal{S}^{\mathcal{B}}\right)-\operatorname{det}\left(\mathcal{S}^{C}\right) \\
\operatorname{tr}\left(\left(\boldsymbol{\Omega}^{\mathcal{A}}\right)^{2} \mathcal{S}^{\mathcal{A}}\right) & =\operatorname{tr}\left(\left(\boldsymbol{\Omega}^{\mathcal{B}}\right)^{2} \mathcal{S}^{\mathcal{B}}\right)+\operatorname{tr}\left(\left(\boldsymbol{\Omega}^{C}\right)^{2} \mathcal{S}^{\mathcal{B}}\right)-\operatorname{det}\left(\boldsymbol{S}^{C}\right)
\end{aligned}
$$

In (18), the strain production is the result of the sum of the normal strain production, the interaction production, and the non-normal production, while, as can be seen from [19], the enstrophy production is the sum of the normal enstrophy production, the interaction production, and the non-normal production.

\section{Governing equations and numerical methods}

In this work, we solve numerically the non-dimensional form of the three-dimensional compressible Navier-Stokes equations as described in Samtaney et al. [7]

$$
\begin{aligned}
& \frac{\partial}{\partial t} \rho+\frac{\partial}{\partial x_{j}}\left(\rho \mathcal{U}_{j}\right)=0, \\
& \frac{\partial}{\partial t}\left(\rho \mathcal{U}_{i}\right)+\frac{\partial}{\partial x_{j}}\left[\rho \mathcal{U}_{i} \mathcal{U}_{j}+\mathcal{P} \delta_{i j} / \gamma \mathrm{Ma}^{2}\right]=\frac{1}{\operatorname{Re}} \frac{\partial}{\partial x_{j}} \sigma_{i j}, \\
& \frac{\partial}{\partial t} \mathcal{E}+\frac{\partial}{\partial x_{j}}\left[\left(\mathcal{E}+\mathcal{P} / \gamma \mathrm{Ma}^{2}\right) u_{j}\right]=\frac{1}{\alpha} \frac{\partial}{\partial x_{j}}\left(\kappa \frac{\partial \mathcal{T}}{\partial x_{j}}\right)+\frac{1}{\operatorname{Re}} \frac{\partial}{\partial x_{j}}\left(\sigma_{i j} \mathcal{U}_{i}\right),
\end{aligned}
$$

where Re, Ma, Pr represent the Reynolds number, the Mach number, and the Prandtl number, respectively. The parameter $\gamma$ is the ratio of the specific heat at constant pressure and the specific heat at constant volume. In the present simulations, the values of $\gamma$ and $\operatorname{Pr}$ are set to 1.4 and 0.7 , respectively. Furthermore, $\alpha$ is defined as $\alpha \equiv \operatorname{Pr} \operatorname{Re}(\gamma-1) \mathrm{Ma}^{2}$.

The primary variables are density, $\rho$, velocity components, $\mathcal{U}_{i}$, temperature, $\mathcal{T}$, and pressure, $\mathcal{P}$. The viscous stress components, $\sigma_{i j}$, and total energy per unit volume, $\mathcal{E}$, are defined by

$$
\begin{aligned}
\sigma_{i j} & \equiv \mu\left(\frac{\partial \mathcal{U}_{i}}{\partial x_{j}}+\frac{\partial \mathcal{U}_{j}}{\partial x_{i}}\right)-\frac{2}{3} \mu \theta \delta_{i j}, \\
\mathcal{E} & \equiv \frac{\mathcal{P}}{(\gamma-1) \gamma M^{2}}+\frac{1}{2} \rho \mathcal{U}_{j} \mathcal{U}_{j}
\end{aligned}
$$

where $\theta=\partial \mathcal{U}_{k} / \partial x_{k}$ is the velocity divergence or dilatation, a variable that quantifies the local rate of rarefaction $(\theta>0)$ or compression $(\theta<0)$. The expressions of the dimensionless dynamical viscosity and thermal conductivity [8] needed to complete the system are as follows:

$$
\mu, \kappa=\frac{c_{1} \mathcal{T}^{3 / 2}}{\mathcal{T}+T_{c}}
$$


where $c_{1}=1.4042$ and $T_{c}=0.4042$.

The presence of shocklets in the flow problem configurations that we solve requires the use of highly accurate numerical solvers capable of capturing shock waves very well. Consequently, the spatial discretization of the divergence of the inviscid fluxes is carried out using a seventh-order accurate hybrid upwinded-WENO scheme Don and Borges [9], whilst the divergence of the viscous fluxes is computed with an eighth-order accurate centered finite difference scheme. Furthermore, the time integration is performed via a third-order accurate total variation diminishing low-storage Runge-Kutta scheme, as described by Gottlieb and Shu [10]).

\section{DNS database}

The decaying isotropic turbulence is an important model to investigate the free compressible turbulence. In this context, it is important to ensure that the turbulence is fully developed.

The present direct numerical simulation is undertaken on a uniform grid with $512^{3}$ points. We carry out a parametric study at various values of the turbulent Mach number, $\mathrm{Ma}_{t}$, for a fixed Taylor micro-scale Reynolds number, $\operatorname{Re}_{\lambda}$, which is set almost to 100 . Table 1 summarizes the one-point statistics of the six flows. The turbulent Mach number, $\mathrm{Ma}_{t}$, ranges from 0.12 to 0.89 . The Kolmogorov length scale is defined as $\eta=\left(\langle\mu / \operatorname{Re}\rangle^{3} /\langle\varepsilon / \rho\rangle\right)^{1 / 4}$, where the kinetic energy dissipation rate per unit volume is defined as $\varepsilon=\sigma_{i j} \mathcal{S}_{i j} /$ Re. The resolution parameter $\Delta x / \eta$ is in the range $1.15 \leq \Delta x / \eta \leq 1.81$, where $\Delta x$ is the grid length in each coordinate direction. In particular, that value of $k_{\max } \eta$, where $k_{\max }$ is the maximum resolved wave number in the grid taken to be equal to half of the number of grid points in each direction, is in the range $2.22<k_{\max } \eta<2.73$. This ensures that the small scales are well resolved in all the cases considered herein [11]. To measure the onset of a realistic turbulence, we use the mean values of velocity derivative skewness, $\mathcal{S} k_{3}$, and flatness, $\mathcal{F} l_{3}$, which are defined as

$$
\begin{gathered}
\mathcal{S} k_{3}=\frac{\left\langle\left(\partial \mathcal{U}_{1} / \partial x_{1}\right)^{3}\right\rangle}{\left\langle\left(\partial \mathcal{U}_{1} / \partial x_{1}\right)^{2}\right\rangle^{3 / 2}}, \\
\mathcal{F} l_{3}=\frac{\left\langle\left(\partial \mathcal{U}_{1} / \partial x_{1}\right)^{4}\right\rangle}{\left\langle\left(\partial \mathcal{U}_{1} / \partial x_{1}\right)^{2}\right\rangle^{2}}
\end{gathered}
$$

From Table 1 , we notice that the magnitude of both $S k_{3}$ and $\mathcal{F} l_{3}$ become larger with the increase of $\mathrm{Ma}_{t}$. This feature is due to the onset of shocklets in a compressible turbulent flow [12]. We also point out that although the thickness of the shocks is comparable to the grid length and is not directly resolved by the WENO scheme, the total amount of dissipation across the shock is independent of numerical viscosity [13].

\section{Results and discussion}

To quantify the statistical properties of compressible turbulent structures with the present velocity gradient tensor decomposition, we formulate the problem in terms of the anisotropic part of the deformation rate tensor, 


\begin{tabular}{cccccccccc}
\hline \hline Resolution & $\mathrm{Re}_{\lambda}$ & $\mathrm{Ma}_{t}$ & $\mathcal{U}^{\prime}$ & $\langle\varepsilon / \rho\rangle$ & $\Delta x / \eta$ & $\mathcal{L}_{t} / \eta$ & $\lambda / \eta$ & $\mathcal{S} k_{3}$ & $\mathcal{F} l_{3}$ \\
\hline \hline $512^{3}$ & 100 & 0.12 & 0.54 & 0.11 & 1.18 & 151 & 19.80 & -0.43 & 5.50 \\
$512^{3}$ & 100 & 0.32 & 0.53 & 0.10 & 1.17 & 154 & 19.79 & -0.45 & 5.64 \\
$512^{3}$ & 100 & 0.50 & 0.53 & 0.10 & 1.15 & 154 & 19.59 & -0.50 & 5.53 \\
$512^{3}$ & 100 & 0.59 & 0.46 & 0.11 & 1.29 & 181 & 19.59 & -0.51 & 5.94 \\
$512^{3}$ & 100 & 0.73 & 0.45 & 0.09 & 1.35 & 175 & 19.37 & -0.71 & 6.10 \\
$512^{3}$ & 100 & 0.89 & 0.45 & 0.07 & 1.41 & 172 & 19.05 & -1.18 & 8.81 \\
\hline \hline
\end{tabular}

Table 1 Flow parameters for DNS simulations

$\mathcal{A}^{*}=\mathcal{A}-\mathcal{S}_{i i}^{\mathcal{A}} \mathcal{I} / 3$. An example of the iso-contour of the magnitude of the components extracted from Schur

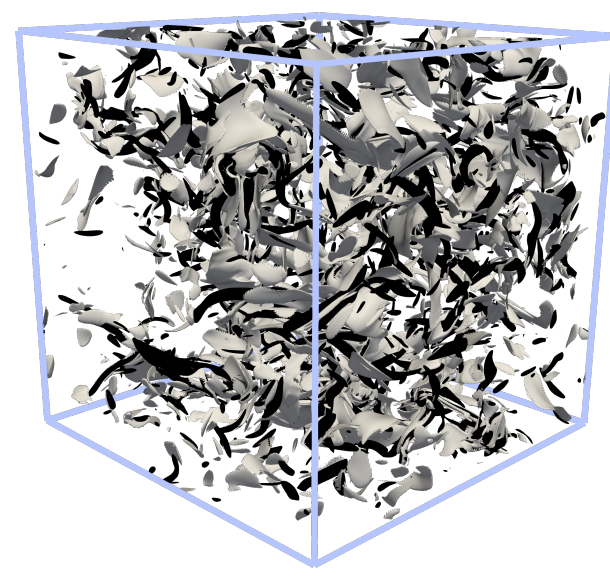

(a) $\left\|\boldsymbol{\Omega}^{\mathcal{B}}\right\|^{2}$ (black), $\left\|\boldsymbol{\Omega}^{C}\right\|^{2}$ (white).

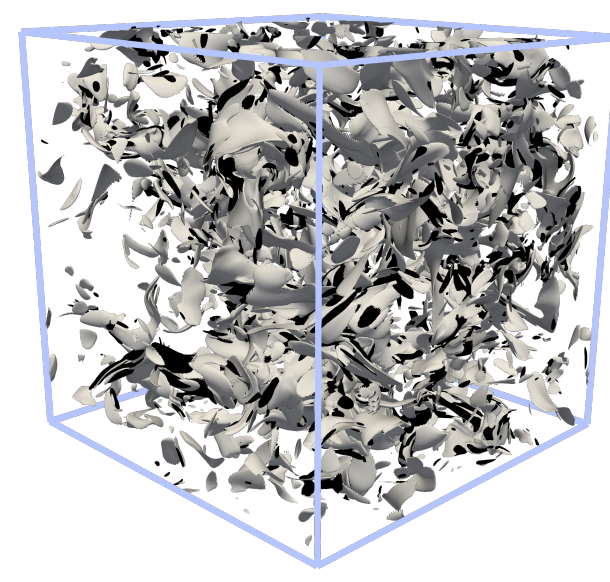

(b) $\left\|\mathcal{S}^{\mathcal{B}}\right\|^{2}$ (black), $\left\|\mathcal{S}^{C}\right\|^{2}$ (white).

Fig. 2 Iso-contour of the components of (15) and (14) for $\mathrm{Ma}_{t}=0.12$.

decomposition are depicted in fig.2 A vortex filament is viewed as a vortex tube of various cross-section for $\left\|\mathbf{\Omega}^{\mathcal{B}}\right\|^{2}$, while the other components exhibit sheet-like structures with higher intermittency for the components of $\left\|\mathcal{S}^{\mathcal{A}}\right\|^{2}$.

We report in fig. 3 the iso-contour lines (of the logarithm) of the second, $Q^{\mathcal{A}}$, and third, $R^{\mathcal{A}}$, invariant at various values of turbulent Mach number, $\mathrm{Ma}_{t}$, of the present database. As expected, all the joint PDFs exhibit the classical tear-drop shape as in the incompressible case, with a statistical preference in the SFS and UNSS quadrants, where statistical points are mostly aligned with the right branch corresponding to $\Delta_{\mathcal{A}^{\star}}=0$. The compression motion tends to reveal a stronger alignment with the right branch, as indicated by a rather sharp joint PDF with the curve $\Delta_{\mathcal{A}^{\star}}=0$ [14]. In the following, the asterisk symbol is omitted for compactness.

\section{A. Estimate of the non-normality effects}

We first estimate the relative importance of the normal and non-normal contributions for the decomposition given by $\mathcal{A}=\mathcal{B}+\mathcal{C}$. We note that for $\|\boldsymbol{C}\| \approx 0$, it is legitimate to model the behavior of $\mathcal{A}$ by using its eigenvalues. The 


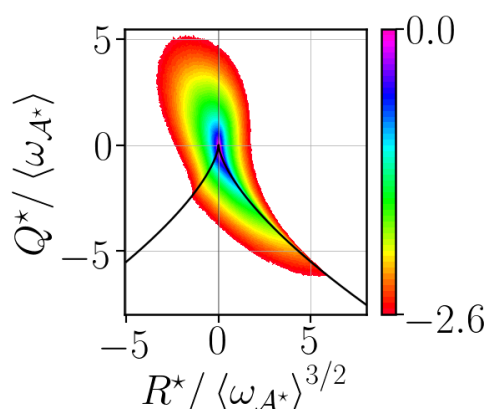

(a) $\mathrm{Ma}_{t}=0.12$

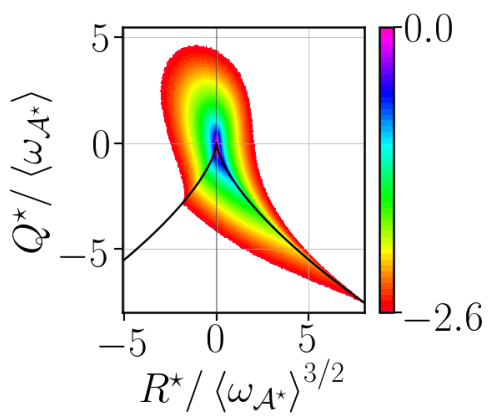

(d) $\mathrm{Ma}_{t}=0.59$

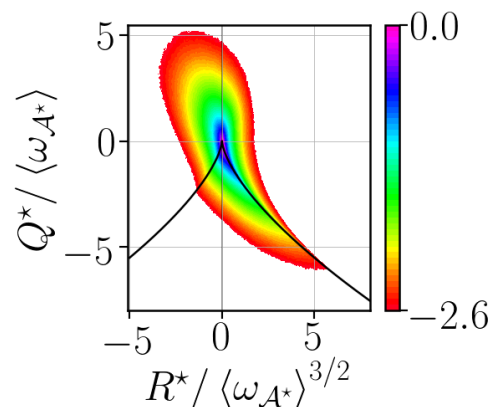

(b) $\mathrm{Ma}_{t}=0.32$

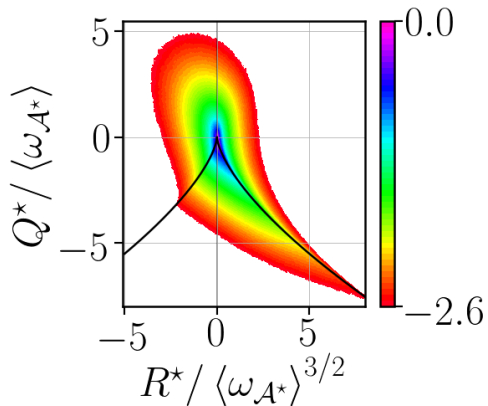

(e) $\mathrm{Ma}_{t}=0.73$

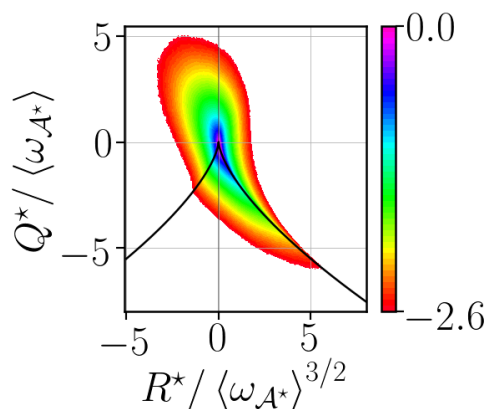

(c) $\mathrm{Ma}_{t}=0.50$

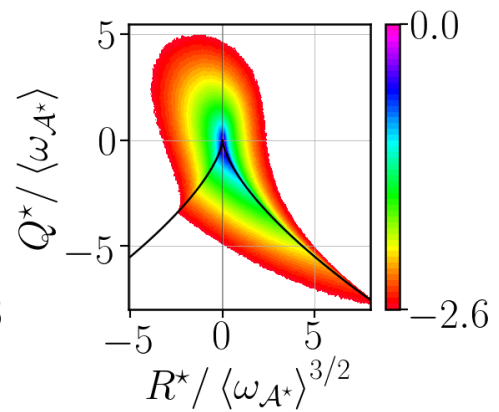

(f) $\mathrm{Ma}_{t}=0.89$

Fig. 3 Joint PDF of $Q$ and $R$ as function of turbulent Mach number.

following standardized difference is used to understand when the non-normality effects have negligible contributions

$$
\kappa_{\mathcal{B}, C}=\frac{\|\mathcal{B}\|-\|\mathcal{C}\|}{\|\mathcal{B}\|+\|C\|}
$$

The normal effects are considered to be important when the values of $\kappa_{\mathcal{B}, C}$ are close to 1 , while they become negligible as $\kappa_{\mathcal{B}, C}$ approaches -1 . In fig. $4 \mathrm{a}$, we show that the overall mode of the distribution of $\kappa_{\mathcal{B}, C}$ is slightly negative, with a median getting close to $\kappa_{\mathcal{B}, C} \approx 0$ as the compressibility intensifies, i.e., the value of turbulent Mach number, $\mathrm{Ma}_{t}$, becomes higher. This result indicates that the non-normality effects (that represent non-local effects) in the dynamics of a homogeneous isotropic turbulent flow are significant to $\mathcal{A}$ as the contributions relative to the eigenvalues. When conditioning the overall PDF of $\kappa_{\mathcal{B}, C}$ by the six regions of joint PDF of $R^{\mathcal{A}}-Q^{\mathcal{A}}$, as depicted in fig. $4 \mathrm{~b}$ and reorganizing the sub-plots by pair associated with a given state of $Q^{\mathcal{A}}$, we observe that the contribution of $\|\boldsymbol{C}\|$ is higher for the left side sub-plots, where $R^{\mathcal{A}}<0$. We note also that the non-normality effects predominantly arise in region 3 , and are less pronounced in region 6. This indicates that a purely eigenvalue-based description of the flow field is effective near the Vieillefosse tail but not in the stable focus region. This behavior is consistent with the findings of Cantwell [15] and Keylock [5]. A higher turbulent Mach number, $\mathrm{Ma}_{t}$, has a tendency to slightly shift the distribution mode of $\kappa_{\mathcal{B}, C}$ to positive values, indicating that the compressibility is likely decreasing the non-normality effects. 


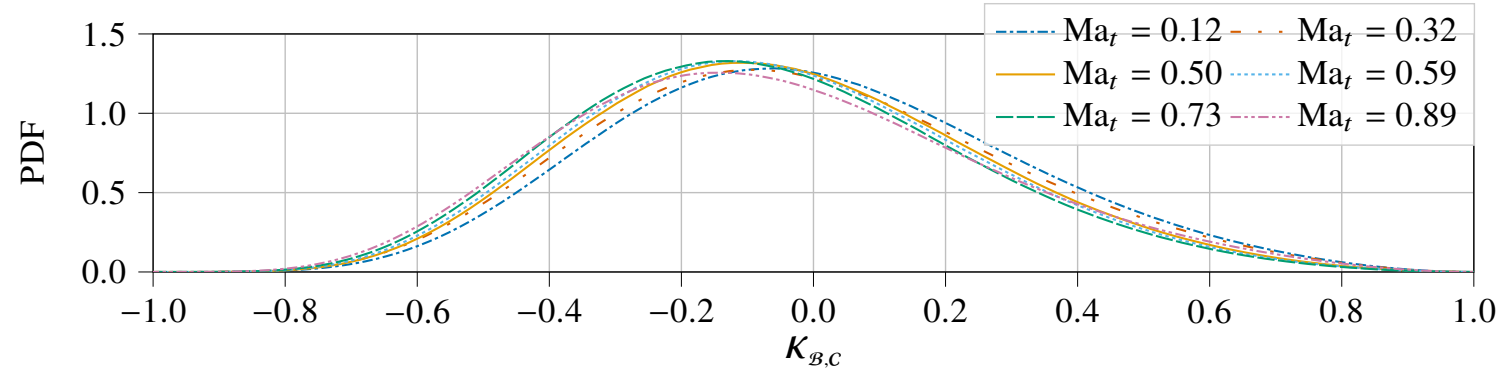

(a)
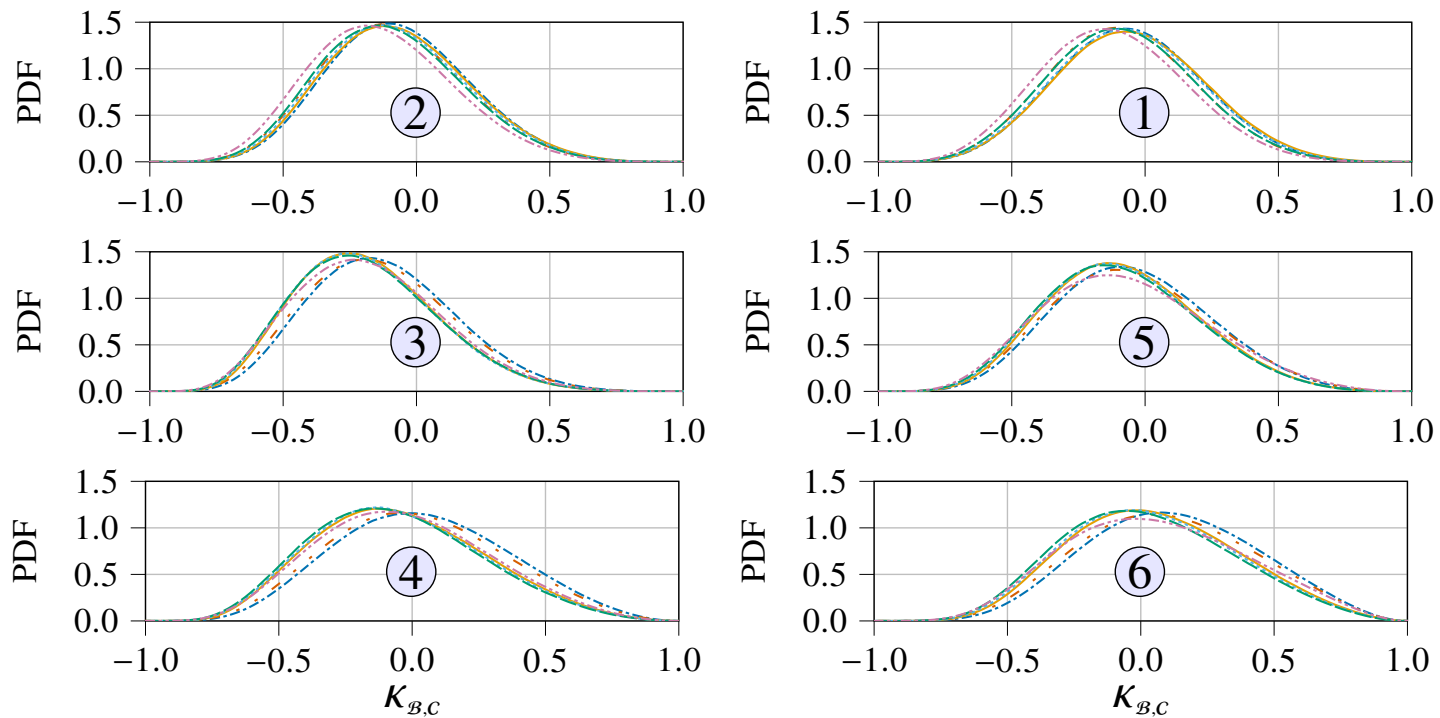

(b)

Fig. 4 PDF of $\kappa_{\mathcal{B}, C}$ shown for all the data and for the DNS database and as a function of the six regions of the $Q^{\mathcal{A}}-R^{\mathcal{A}}$ diagram in the various panels.

\section{B. Estimate of non-normality effects on the second invariant $R^{\mathcal{A}}$}

We used again a standardized difference to evaluate the importance of non-normality effects on the enstrophy and the dissipation as given in 160 :

$$
\kappa_{\mathcal{B}, C}^{\mathbf{\Omega}^{\mathcal{A}}}=\frac{\left\|\boldsymbol{\Omega}^{\mathcal{B}}\right\|-\left\|\boldsymbol{\Omega}^{C}\right\|}{\left\|\mathbf{\Omega}^{\mathcal{B}}\right\|+\left\|\boldsymbol{\Omega}^{C}\right\|},
$$

and

$$
\kappa_{\mathcal{B}, C}^{\mathcal{S}^{\mathcal{P}}}=\frac{\left\|\mathcal{S}^{\mathcal{B}}\right\|-\left\|\mathcal{S}^{C}\right\|}{\left\|\mathcal{S}^{\mathcal{B}}\right\|+\left\|\mathcal{S}^{C}\right\|} .
$$

Since $\kappa_{\mathcal{B}, C}^{\Omega^{\mathcal{A}}}=-1$ for $\Delta^{\mathcal{A}}<0$, the results for $\boldsymbol{\kappa}_{\mathcal{B}, C}^{\Omega^{\mathcal{H}}}$ are not shown in the sub-plots and are excluded from the overall PDF. As can be seen in fig. $5 \mathrm{~b}$, the distribution mode of $\kappa_{\mathcal{B}, C}^{\Omega^{\mathcal{A}}}$ (resp. $\kappa_{\mathcal{B}, C}^{\mathcal{S}^{\mathcal{H}}}$ ) increases (resp. decreases) by increasing the values of $Q^{\mathcal{A}}$ and increases with increasing the values of $R^{\mathcal{A}}$. In the non-focal regions, the dynamic of the rotation-rate tensor seems to be dictated only by the non-normal part, $\|C\|$, since $\kappa_{\mathcal{B}, C}^{\Omega^{\mathcal{P}}} \approx-1$. Note that the $\kappa_{\mathcal{B}, C}^{\mathcal{S}^{\mathcal{P}}}$ associated with the current database has a distinctive mode distribution at $\kappa_{\mathcal{B}, C}^{\mathcal{S}^{\mathcal{P}}} \approx-0.2$, which is not observed in the Johns Hopkins database as post-processed by Keylock [5]. All these observed trends are intensified by the effect of the compressibility, which tends 
to shift, for instance, the mode distribution of $\kappa_{\mathcal{B}, C}^{\mathcal{S}^{\mathcal{P}}}$ to more positive values. Thus, the non-normal effects are significantly important in the vortical regions, i.e., region 1 and 2. This suggests that the vortical properties in these regions should include the non-normal contributions.

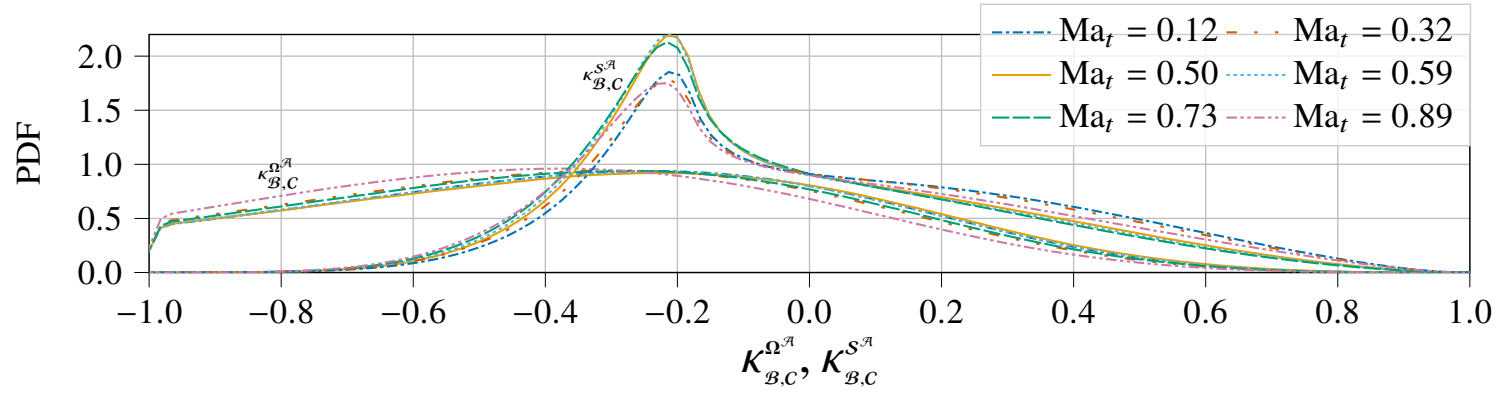

(a)
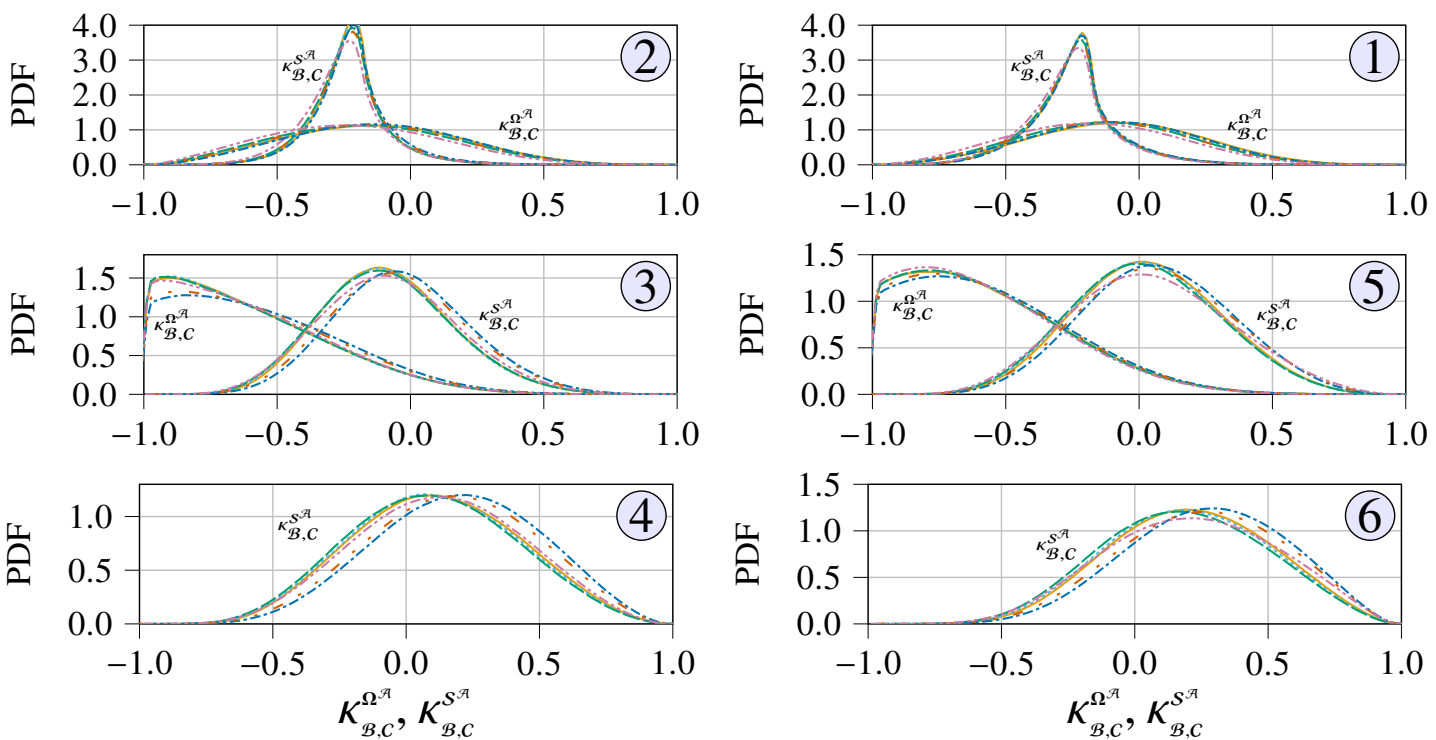

(b)

Fig. 5 PDF of $\kappa_{\mathcal{B}, C}^{\mathcal{S}^{\mathcal{P}}}$ and $\kappa_{\mathcal{B}, C}^{\Omega^{\mathcal{F}}}$ shown for all the data and for the DNS database and as a function of the six regions of the $Q^{\mathcal{A}}-R^{\mathcal{A}}$ diagram in the various panels. In the overall PDF of $\kappa_{\mathcal{B}, C}^{\mathbf{\Omega}^{\mathcal{A}}}$, the data corresponding to $\kappa_{\mathcal{B}, C}^{\mathbf{\Omega}^{\mathcal{A}}} \approx=-1$ associated to regions 4 and 6 are excluded.

\section{Joint PDF of $Q^{\mathcal{S}^{\mathcal{B}}}$ and $Q^{\mathcal{S}^{C}}$}

Additional insights on the effect of the non-normality can be gleaned from the joint PDF of $Q^{\mathcal{S}^{\mathcal{B}}}$ and $Q^{\mathcal{S}^{C}}$. In fig. 6 . we depict the joint PDF of $Q^{\mathcal{S}^{\mathcal{B}}}$ and $Q^{\mathcal{S}^{C}}$ normalized by the magnitude of the enstrophy, $\left\langle\omega_{\mathcal{A}}\right\rangle$. We observe that $Q^{\mathcal{S}^{C}}$ is practically always negative and this seems to be independent from the compressibility. However, the distribution of $Q^{\mathcal{S}^{\mathcal{B}}}$ is skewed towards negative values and this, contrary to what we observe for $Q^{\mathcal{S}^{C}}$, is likely associated with the intensity of the compressibility effects. The probability of $Q^{\mathcal{S}^{\mathcal{B}}}$ reaches its maximum value at the highest turbulent Mach number, $\mathrm{Ma}_{t}=0.89$, indicating that the compressibility mainly acts on the distribution of the normal part of the velocity gradient tensor, $\mathcal{B}$. 


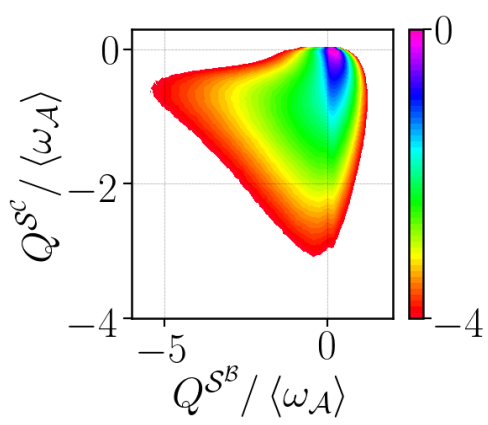

(a) $\mathrm{Ma}_{t}=0.12$

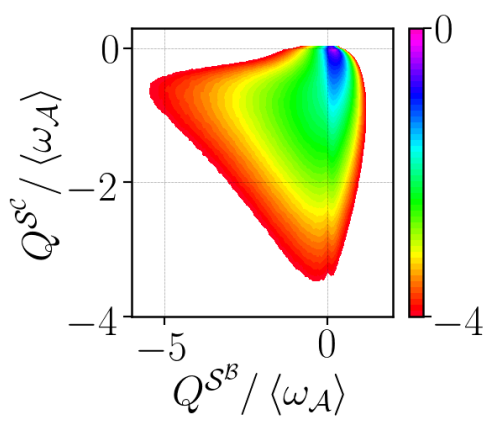

(d) $\mathrm{Ma}_{t}=0.59$

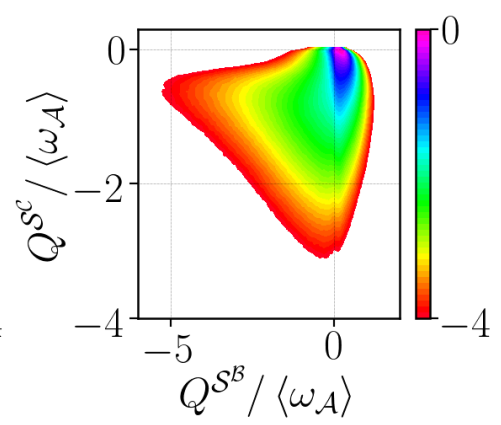

(b) $\mathrm{Ma}_{t}=0.32$

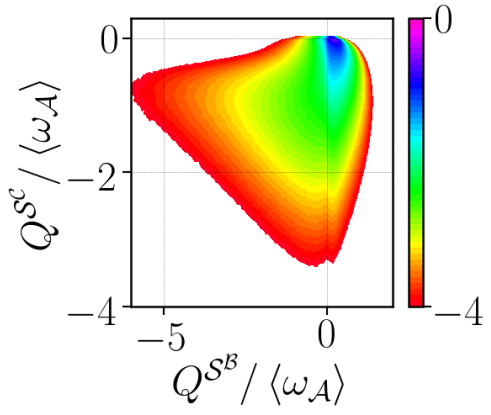

(e) $\mathrm{Ma}_{t}=0.73$

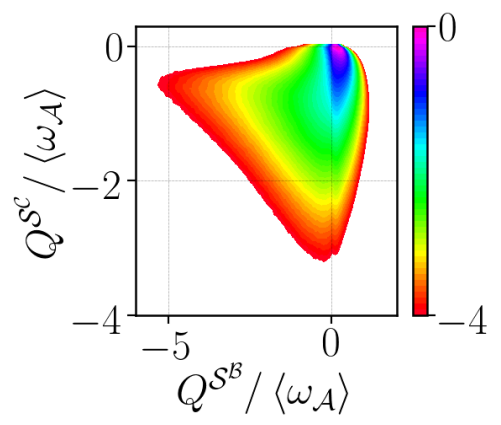

(c) $\mathrm{Ma}_{t}=0.50$

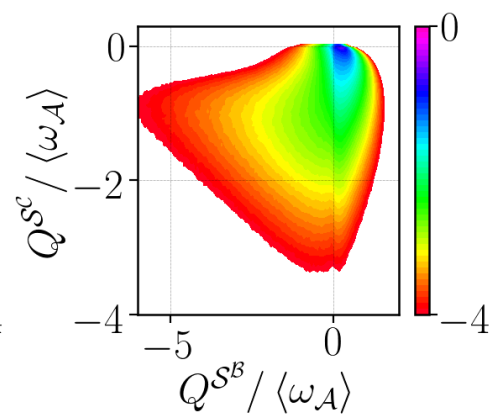

(f) $\mathrm{Ma}_{t}=0.89$

Fig. 6 Joint PDF of $Q^{\mathcal{S}^{\mathcal{B}}}$ and $Q^{\mathcal{S}^{C}}$ normalized by $\left\langle\omega_{\mathcal{A}}\right\rangle$ as function of turbulent Mach number.

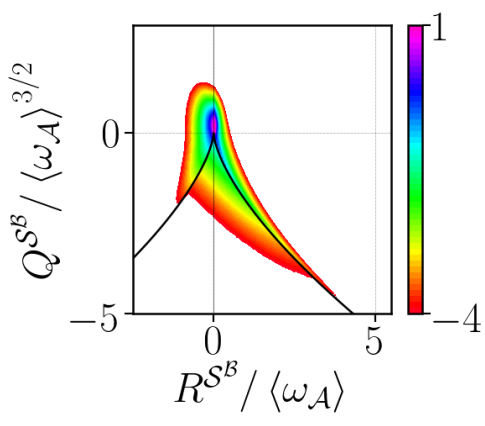

(a) $\mathrm{Ma}_{t}=0.12$

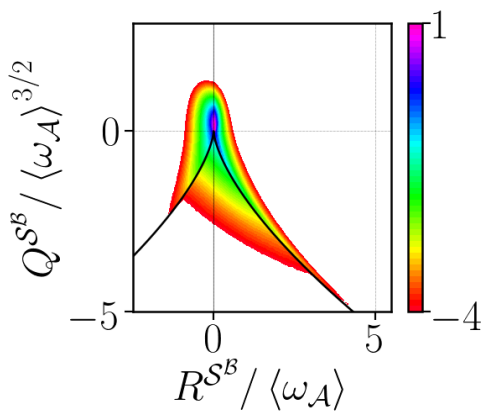

(d) $\mathrm{Ma}_{t}=0.59$

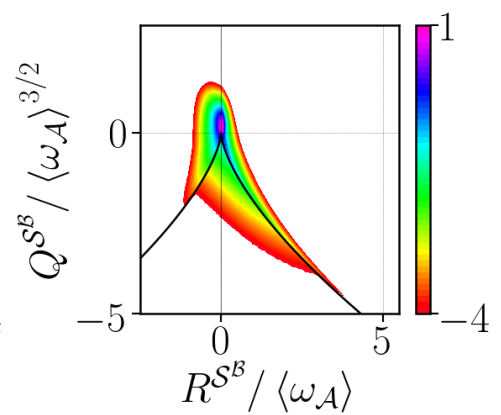

(b) $\mathrm{Ma}_{t}=0.32$

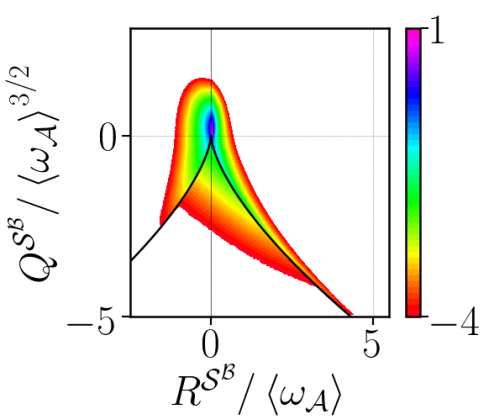

(e) $\mathrm{Ma}_{t}=0.73$

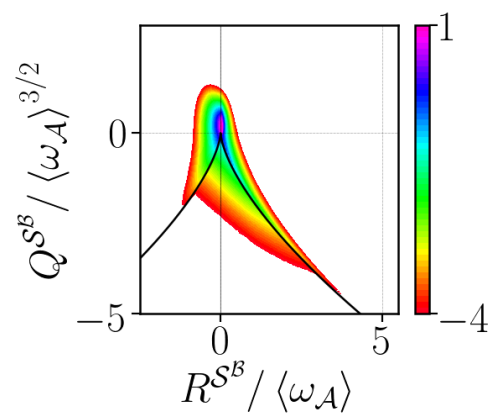

(c) $\mathrm{Ma}_{t}=0.50$

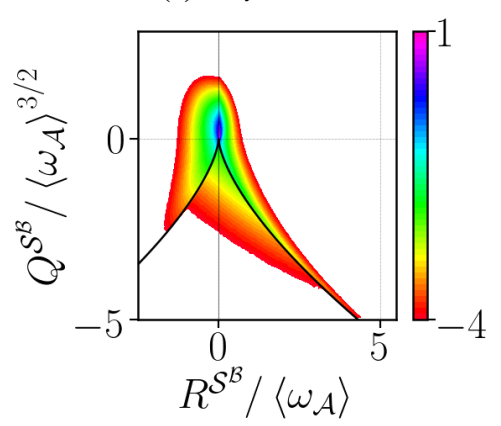

(f) $\mathrm{Ma}_{t}=0.89$

Fig. 7 Joint PDF of $R^{\mathcal{S}^{\mathcal{B}}}$ and $Q^{\mathcal{S}^{\mathcal{B}}}$ normalized by $\left\langle\omega_{\mathcal{A}}\right\rangle$ as function of turbulent Mach number. 


\section{Joint PDF of $R^{\mathcal{S}^{\mathcal{B}}}$ and $Q^{\mathcal{S}^{\mathcal{B}}}$}

The joint PDF of the normalized $R^{\mathcal{S}^{\mathcal{B}}}$ and $Q^{\mathcal{S}^{\mathcal{B}}}$ are shown in fig. 7 for different turbulent Mach number, $\mathrm{Ma}_{t}$. We note that the shapes of the joint PDFs are related to the intensity of the compressibility of the flow field. In comparison with the classical joint PDF of $R^{\mathcal{A}}$ and $Q^{\mathcal{A}}$, the joint PDF related to the normal part of the strain-rate tensor, $\mathcal{S}^{\mathcal{B}}$, is associated with the flow field properties in such a way that the teardrop shape around the origin and Vieillefosse tail are in the stable focus stretching local topology (regions 2 and 3). Furthermore, we observe that independently of the turbulent Mach number, $\mathrm{Ma}_{t}$, the joint PDFs are symmetric with respect to $R^{\mathcal{A}}=0$ for $Q^{\mathcal{A}}>0$, and have a long tail along both the $R^{\mathcal{A}}<0$ and $R^{\mathcal{A}}>0$ parts of the discriminant curve $\Delta^{\mathcal{A}}=0$, as $\mathrm{Ma}_{t}$ increases. Moreover, we notice that the joint PDFs of $R^{\mathcal{S}^{\mathcal{B}}}$ and $Q^{\mathcal{S}^{\mathcal{B}}}$ exhibit a short tail along the $R^{\mathcal{A}}<0$ part of curve $\Delta^{\mathcal{A}}=0$, compared to the joint PDFs of the $R^{\mathcal{A}}$ and $Q^{\mathcal{A}}$ invariants.

\section{E. Nature of the straining in the velocity gradient tensor}

Here we analyze the influence of the compressible intensity in further detail by considering the statistics of the second eigenvalue of the strain rate (or intermediate principal eigenvalue), which is known to i) reflect the intensity of the turbulent transfer, and ii) provide insightful information on the structure of the strain rate field [3, 16]. The strain state acting on each region of the diagram $R^{\mathcal{A}}-Q^{\mathcal{A}}$ is interpreted using the normalization of the intermediate eigenvalue parameter [16],

$$
e_{\mathcal{A}}=\frac{3 \sqrt{6} R^{\mathcal{S}^{\mathcal{A}}}}{\left(-2 Q^{\mathcal{S}^{\mathcal{A}}}\right)^{3 / 2}} .
$$

This parameter ranges from -1 to 1 and compares the magnitudes of the three strain-rate eigenvalues $\lambda_{i}$ by taking into account that they must add up to zero. Furthermore, this quantity allows to estimate the local deformations that cause the strain-rate tensor. An axisymmetric extension and an axisymmetric contraction occur when $e_{\mathcal{A}}=1$ and $e_{\mathcal{A}}=-1$, respectively. In their DNS data, Lund and Rogers [16] found that the most probable case in the HIT test case is the axisymmetric extension and that this state is more correlated with regions of high dissipation. Similarly to Lund and Rogers [16], we analyze the state of strain due to normal and non-normal contributions acting on the flow field by studying the following parameters [5]:

$$
e_{\mathcal{B}}=\frac{3 \sqrt{6} R^{\mathcal{S}^{\mathcal{B}}}}{\left(-2 Q^{\mathcal{S}^{\mathcal{H}}}\right)^{3 / 2}}, \quad e_{C}=\frac{3 \sqrt{6} R^{\mathcal{S}^{C}}}{\left(-2 Q^{\mathcal{S}^{\mathcal{H}}}\right)^{3 / 2}}
$$

Since $R^{\mathcal{S}^{\mathcal{A}}}=R^{\mathcal{S}^{\mathcal{B}}}+R^{\mathcal{S}^{C}}+\operatorname{tr}\left(\left(\mathbf{\Omega}^{C}\right)^{2} \mathcal{S}^{\mathcal{B}}\right)$, then $e_{\mathcal{A}}=e_{\mathcal{B}}+e_{C}+e_{C, \mathcal{B} \mid \mathcal{A}}$, where the normal straining of non-normality metric is defined as

$$
e_{C, \mathcal{B} \mid \mathcal{A}}=\frac{3 \sqrt{6} \operatorname{tr}\left(\left(\boldsymbol{\Omega}^{C}\right)^{2} \mathcal{S}^{\mathcal{B}}\right)}{\left(-2 Q^{\mathcal{S}^{\mathcal{A}}}\right)^{3 / 2}} .
$$


In fig. 8, we report the PDF of $e_{\mathcal{A}}$ for all the cases of our DNS database. As expected, we observe that the turbulent velocity gradients are biased toward $e_{\mathcal{A}}>0$ and peak strongly at $e_{\mathcal{A}} \approx 1$, meaning that the state of the straining axisymmetric extension forms a disk-like structure [17]. We also note that the peak at 1 is higher for the incompressible case. When subdividing the results by regions of the $Q^{\mathcal{A}}-R^{\mathcal{A}}$ diagram as shown in fig. 9 , we note that the tendency

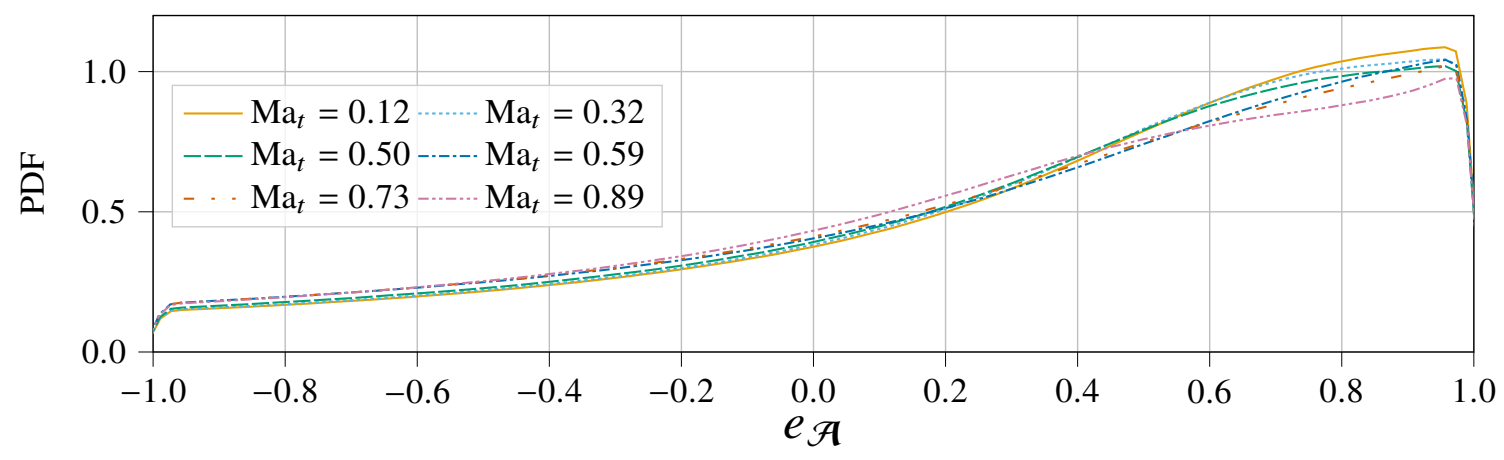

Fig. 8 PDF of $e_{\mathcal{A}}$ for all the turbulent Mach number of the present DNS database

observed in fig. 8 is mainly driven by the regions 2,5 and 6. Moreover, when looking at the profiles of $e_{\mathcal{A}}$ conditioned by $\kappa_{\mathcal{B}, \mathcal{C}}^{\mathcal{S}^{\mathfrak{P}}}>0$, we get, as expected, in region $1, e_{\mathcal{A}} \approx 1$ and, in region $2, e_{\mathcal{A}} \approx-1$. However, note that the states conditioned by $\kappa_{\mathcal{B}, C}^{\mathcal{S}^{\mathcal{P}}}<0$ arise more frequently and they are mostly impacted by the effect of dilatation. Consequently, the non-normal contribution to the strain tensor is important and acts in a different way than the normal part based on the eigenvalues of the velocity gradient tensor.

\section{Conclusion}

In this work, we investigate the small-scale structures of compressible turbulence using a recently additive decomposition of the velocity gradient tensor, $\mathcal{A}$, into normal and non-normal tensors, $\mathcal{B}$ and $\mathcal{C}$. We perform a set of direct numerical simulations of decaying compressible turbulence at six turbulent Mach numbers between $\mathrm{M}_{t}=0.12$ and $\mathrm{M}_{t}=0.89$ and a Reynolds number based on the Taylor micro-scale of $\operatorname{Re}_{\lambda}=100$. The initial field is generated using a physically consistent initialization of compressible turbulence, where there are finite density, temperature, and dilatation fluctuations. The findings of this work are mainly in the difficulty of applying a pure eigenvalue-focused approach to analyze the dynamics of complex systems such a turbulence. In particular, the complex behavior in regions 1,2 , and 3 in $R^{\mathcal{A}}-Q^{\mathcal{A}}$ space demonstrates the difficult task of of understanding the non-local effects without taking into account both the eigenvalues and the non-normal contributions to the velocity gradient tensor. We must caution though, that we consider these findings preliminary, and in particular we note that the results may not be valid for higher turbulent Mach numbers. 

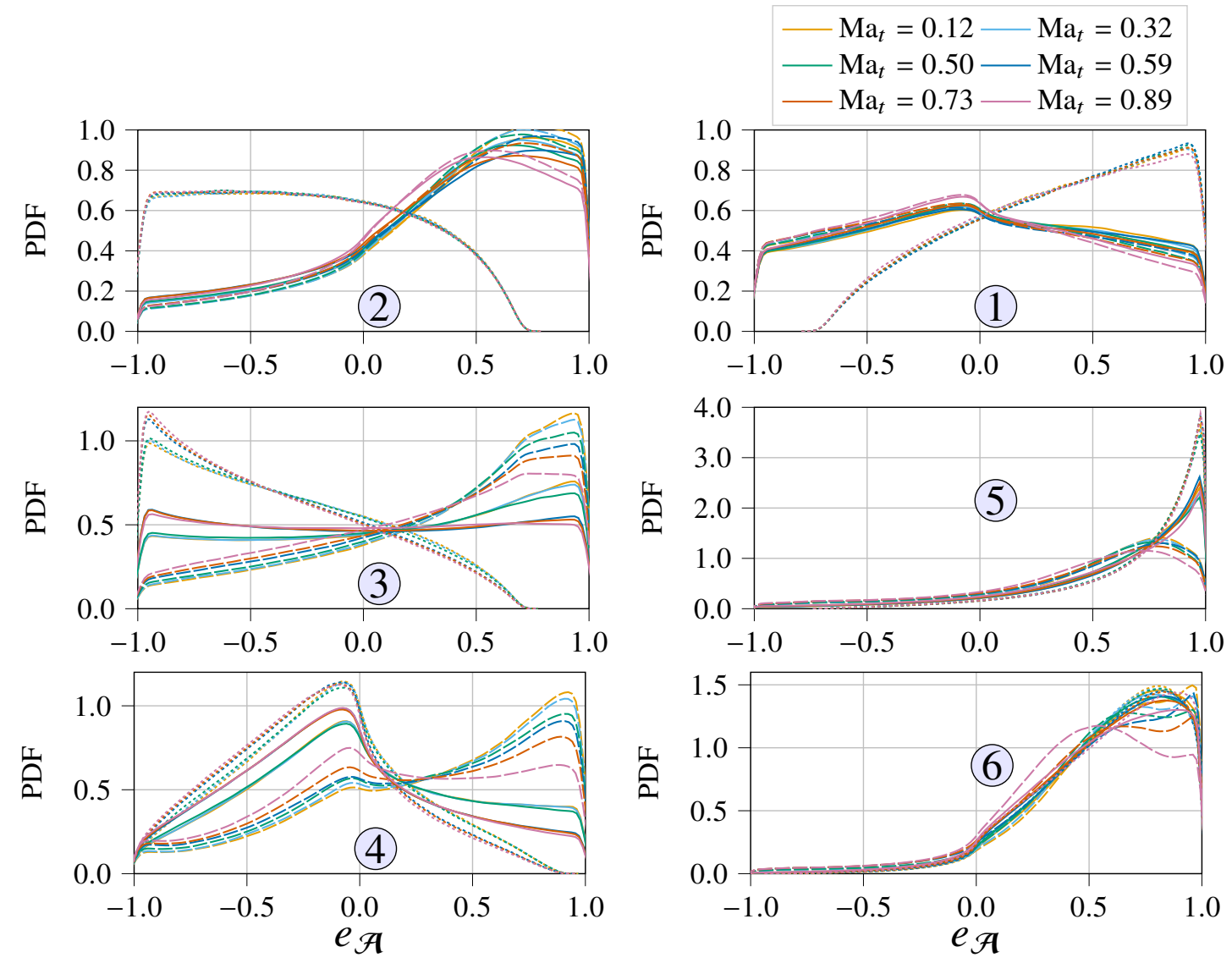

Fig. 9 PDF of $e_{\mathcal{A}}$ for all the turbulent Mach numbers of the present DNS database by each region of the $Q^{\mathcal{A}}-R^{\mathcal{A}}$ diagram (solid line). Results are also shown conditioned on the sign of $\kappa_{\mathcal{B}, C}^{S^{\mathcal{A}}}$, with $\kappa_{\mathcal{B}, C}^{\mathcal{S}^{\mathcal{A}}}>0$ with dotted lines and $\kappa_{\mathcal{B}, C}^{\mathcal{S}}<0$ with dashed lines.

\section{Acknowledgments}

The research reported in this paper was funded by King Abdullah University of Science and Technology. We are thankful for the computing resources of the Supercomputing Laboratory and the Extreme Computing Research Center at King Abdullah University of Science and Technology.

\section{References}

[1] Chong, M. S., Perry, A. E., and Cantwell, B. J., "A general classification of three-dimensional flow fields," Physics of Fluids A: Fluid Dynamics, Vol. 2, No. 5, 1990, pp. 765-777.

[2] Suman, S., and Girimaji, S. S., "Velocity gradient invariants and local flow-field topology in compressible turbulence," Journal of Turbulence, Vol. 11, 2010, p. N2.

[3] Boukharfane, R., Bouali, Z., and Mura, A., "Evolution of scalar and velocity dynamics in planar shock-turbulence interaction," Shock Waves, Vol. 28, No. 6, 2018, pp. 1117-1141. 
[4] Beaumard, P., Buxton, O. R. H., and Keylock, C. J., "The importance of non-normal contributions to velocity gradient tensor dynamics for spatially developing, inhomogeneous, turbulent flows," Journal of Turbulence, Vol. 20, No. 9, 2019, pp. 577-598.

[5] Keylock, C. J., "The Schur decomposition of the velocity gradient tensor for turbulent flows," Journal of Fluid Mechanics, Vol. 848, 2018, pp. 876-905.

[6] Schur, I., "Über die charakteristischen Wurzeln einer linearen Substitution mit einer Anwendung auf die Theorie der Integralgleichungen," Mathematische Annalen, Vol. 66, No. 4, 1909, pp. 488-510.

[7] Samtaney, R., Pullin, D. I., and Kosović, B., "Direct numerical simulation of decaying compressible turbulence and shocklet statistics," Physics of Fluids, Vol. 13, No. 5, 2001, pp. 1415-1430.

[8] Sutherland, W., "The viscosity of gases and molecular force," The London, Edinburgh, and Dublin Philosophical Magazine and Journal of Science, Vol. 36, No. 223, 1893, pp. 507-531.

[9] Don, W.-S., and Borges, R., "Accuracy of the weighted essentially non-oscillatory conservative finite difference schemes," Journal of Computational Physics, Vol. 250, 2013, pp. 347-372.

[10] Gottlieb, S., and Shu, C.-W., "Total variation diminishing Runge-Kutta schemes," Mathematics of Computation, Vol. 67, No. 221, 1998, pp. 73-85.

[11] Jiménez, J., Wray, A. A., Saffman, P. G., and Rogallo, R. S., "The structure of intense vorticity in isotropic turbulence,” Journal of Fluid Mechanics, Vol. 255, 1993, pp. 65-90.

[12] Wang, J., Wan, M., Chen, S., and Chen, S., "Kinetic energy transfer in compressible isotropic turbulence," Journal of Fluid Mechanics, Vol. 841, 2018, pp. 581-613.

[13] Zhang, Y.-T., Shi, J., Shu, C.-W., and Zhou, Y., "Numerical viscosity and resolution of high-order weighted essentially nonoscillatory schemes for compressible flows with high Reynolds numbers," Physical Review E, Vol. 68, No. 4, 2003, p. 046709.

[14] Wang, J., Shi, Y., Wang, L.-P., Xiao, Z., He, X., and Chen, S., "Effect of compressibility on the small-scale structures in isotropic turbulence," Journal of Fluid Mechanics, Vol. 713, 2012, pp. 588-631.

[15] Cantwell, B. J., "Exact solution of a restricted Euler equation for the velocity gradient tensor," Physics of Fluids A: Fluid Dynamics, Vol. 4, No. 4, 1992, pp. 782-793.

[16] Lund, T. S., and Rogers, M. M., "An improved measure of strain state probability in turbulent flows," Physics of Fluids, Vol. 6, No. 5, 1994, pp. 1838-1847.

[17] Meneveau, C., "Lagrangian dynamics and models of the velocity gradient tensor in turbulent flows," Annual Review of Fluid Mechanics, Vol. 43, 2011, pp. 219-245. 